\title{
Magnetic Resonance T2 Relaxometry In Knee Joint Patellar Cartilage Imaging
}

\author{
${ }^{1}$ Raj Kumar Soundarajan, ${ }^{2}$ Rajeswaran $\mathbf{R}$ and ${ }^{3}$ Sheila Elangovan \\ ${ }^{1}$ Aster Hospital, DM Health group, Dubai, UAE \\ ${ }^{2,3}$ College of Allied Health Sciences, Department of Radiology and Imaging sciences, \\ Sri Ramachandra University, Chennai, India \\ ${ }^{1}$ rajpmakumar@icloud.com
}

\begin{abstract}
Background: Over the past decade the diagnosis of knee disorders has improved significantly through better imaging techniques. Imaging of the Knee joint includes Radiograph, Computed Tomography, Magnetic Resonance Imaging, Arthrography and of advanced imaging techniques like T2 Relaxometry.

Aim: To obtain T2 relaxometry value of knee joint patellar cartilages \& to compare the T2 relaxometry values of the Osteoarthritic patients with that of the other cause.

Material \& Methods: 20 patients who presented themselves in Radiology department of either sex whose reports and image data's are collected prospectively during the study period of December 2011 to February 2012. All the patients' data within the study period were collected. Patients were selected irrespective of their age group, gender and pathologic findings, a detailed history with various patient's data includes patient demography, age, sex and the study reports are collected and is entered in a specially designed Profoma. The acquired study data of Sagittal T2 Mapping High Resolution sequence of each patient are then post processed by using a GE Advantage Workstation (version 4.4) and T2 Relaxometry values of various knee joint cartilages (Medial Patellar cartilage, Lateral Patellar cartilage) are collected by using a special software and is entered in the Profoma table.
\end{abstract}

Conclusion: Conventional MRI may not show early cartilage changes; Cartilage edema following trauma (or) Due to osteoarthritis can be picked up early by T2 Mapping. hence it is useful in early patient management.

Key words: MRI, Knee joint imaging, Patellar Imaging, T2 Relaxometry, T2 Mapping, osteoarthritis, Knee trauma.

\section{Introduction}

Over the past decade the diagnosis of knee disorders has improved significantly through better imaging techniques. Imaging of the Knee joint includes Plain Radiograph, Computed Tomography (CT), Magnetic Resonance Imaging (MRI), Arthrography and of advanced imaging techniques like T2 Relaxometry. Radiographs of the knee are usually the first imaging studies, especially if bone wear and tear is suspected clinically. Radiographs are often required after acute trauma. The most common views are 
Raj Kumar Soundarajan, Rajeswaran R and Sheila Elangovan; Magnetic Resonance T2 Relaxometry In Knee Joint Patellar Cartilage Imaging. Journal of Biomedical Engineering and Medical Imaging, Volume 3, No 5, October (2016), pp 18-28

the Antero Posterior (AP), the Lateral, and the Skyline view. Early changes in the cartilage and other articular tissues are not directly visible. In Computed Tomography, axial images were obtained and can be reformatted into sagittal and coronal planes. It also allows 3 dimensional (3D) reconstruction of knee joint and is very useful in case of trauma, fractures, etc. It provides good details about bony structures with less tissue contrast when compared to that of MRI.

\subsection{Magnetic Resonance Imaging:}

MR imaging is a powerful tool for the morphologic and compositional imaging of cartilage in the knee. MRI offers multi-planar capabilities, high spatial resolution without ionizing radiation, and superior contrast between joint tissues. For routine clinical examinations of the knee joint, most departments are still applying a multiplane 2D fast SE sequence, alone or in combination with a 3D GRE sequence to improve cartilage assessment. The combined use of high-resolution morphologic imaging techniques and compositional imaging techniques may lead to increased sensitivity of MR imaging for the detection of early cartilaginous degeneration and increased utility for the assessment of cartilage repair techniques.

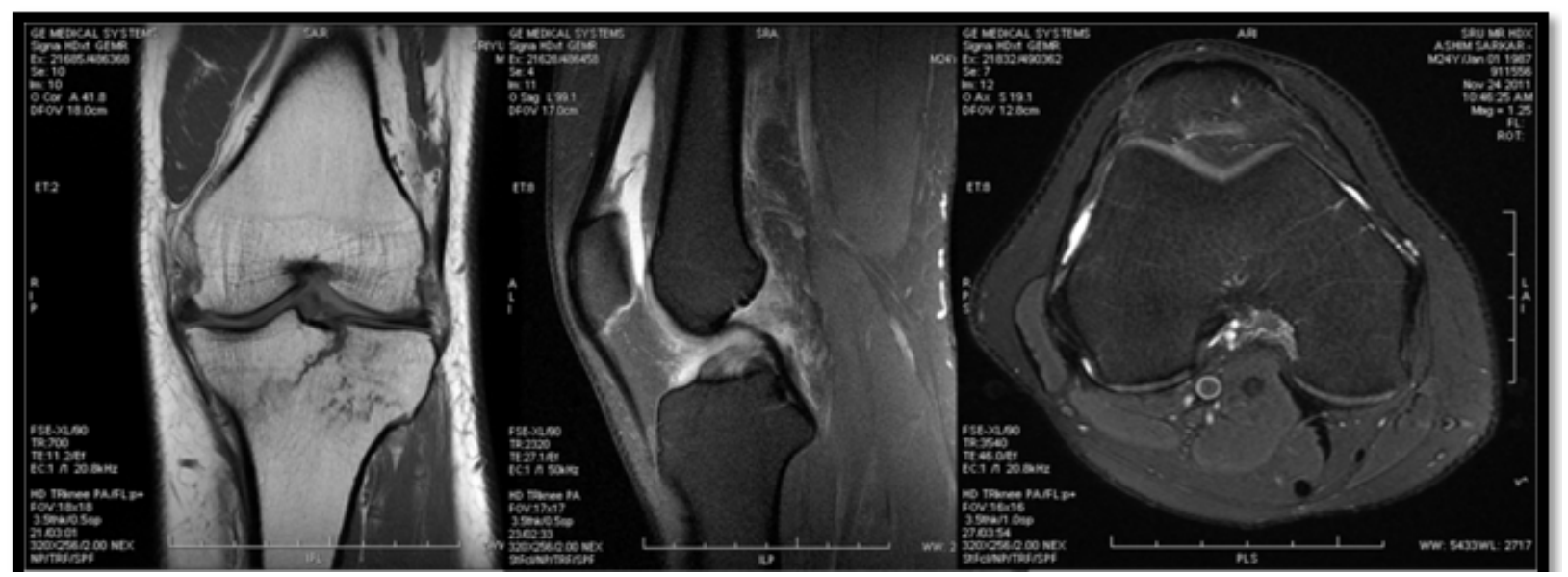

Figure 1: MRI - Coronal T1 / Sagittal /Axial Proton Density with Fat Suppression

\subsection{T2 Mapping:}

T2 relaxation time is a non-invasive marker of cartilage degeneration because it is sensitive to tissue hydration and biochemical composition. When collagen breaks down, there is increased mobility of water in the cartilage and therefore a prolongation in T2 relaxation times. It is based on a multi-echo pulse sequence derived from the existing FSE-XL that can create up to 8 echoes per single acquisition not more than eight echoes are acquired, due to the cartilage short $\mathrm{T} 2$ relaxation times. Cartigram automatically generates color-maps based on a scale of $\mathrm{T} 2$ values that allows visualization of changes in the composition of articular cartilage in some cases before changes in the thickness can be seen. 


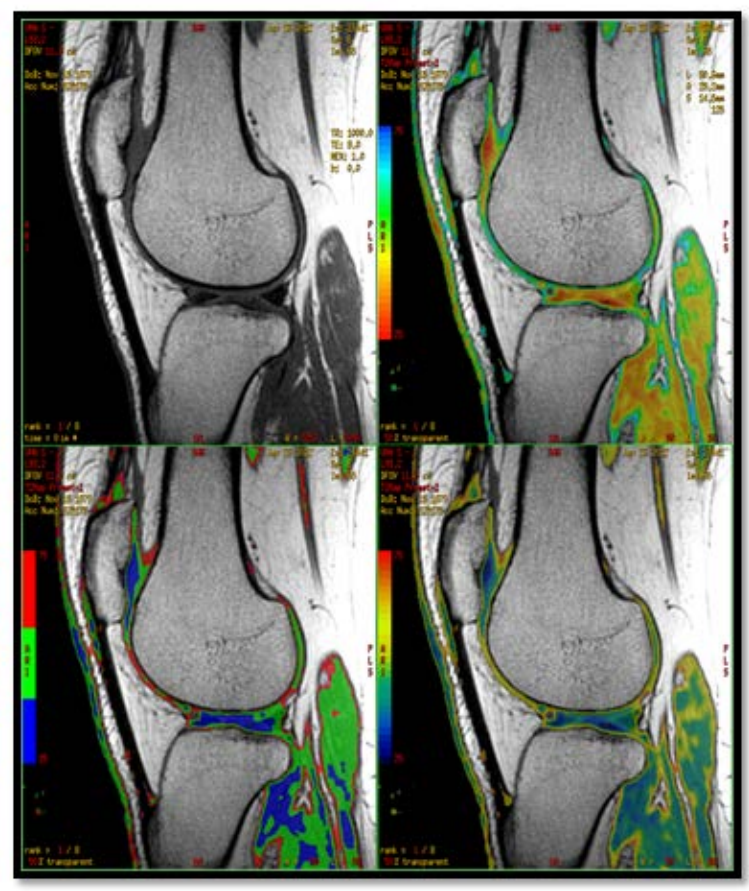

Figure 2: MR-T2 Mapping Sagittal plane

\section{AIM}

- To obtain T2 relaxometry value of knee joint patellar cartilages

- To compare the T2 relaxometry values of the Osteoarthritic patients with that of the other cause.

\section{Anatomy of The Knee}

The knee joint is the largest synovial joint in the body. It consists of the articulation between the femur and tibia, which is weight bearing; and the articulation between the patella and the femur, which allows the pull of the quadriceps femoris muscle to be directed anteriorly over the knee to the tibia without tendon wear. two fibrocartilaginous menisci, one on each side, between the femoral condyles and tibia accommodate the changes in the shape of the articular surfaces during joint movements. The detailed movements of the knee joint are complex, but basically the joint is a hinge joint that allows mainly flexion and extension.

The major cartilages associated with the knee joint are 1, The Patellar Cartilage, 2, The Collateral Cartilages \& 3, The Cruciate Cartilages.

\subsection{Patellar Cartilage}

The Patellar cartilage is basically the continuation of the quadriceps femoris tendon inferior to the patella. It is attached above to the margins and apex of the patella and below to the tibial tuberosity. 


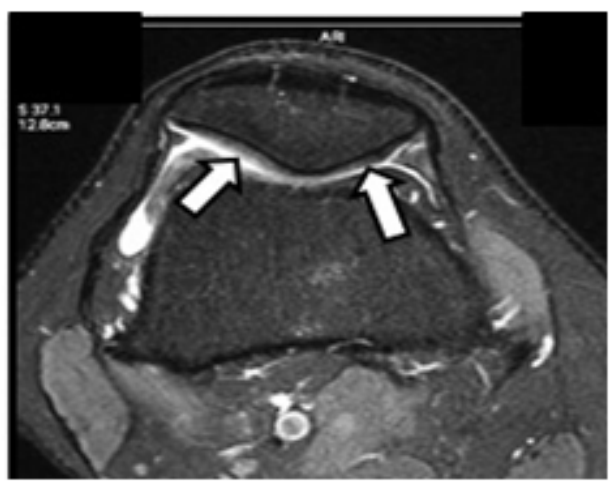

Figure 3: MRI Axial Image shows cross section of patellar cartilage (white arrows)

\section{PROTOCAL - MRI OF KNEE}

Exclusive MRI screened has done to all patients with metal detector before the procedure to avoid complications due to any implants. Possible contra-indications must be checked. Metallic jewelers should be removed prior to MR examinations as it because RF burns due to high RF absorption locally; The MR imaging sequences most commonly used in the assessment of joint cartilage are 2D or multisection T1weighted, proton density-weighted and T2-weighted imaging sequences with or without fat suppression.

4.1 PATIENT POSITIONING: Patient was made to lay down in Supine position with The knee in the center of the coil with the external rotation of $15^{\circ}$ to $20^{\circ}$, flexed to $5^{0}$ to $10^{0}$ and the coil was closed after adequate immobilization and the landmark was set in the center of the coil.

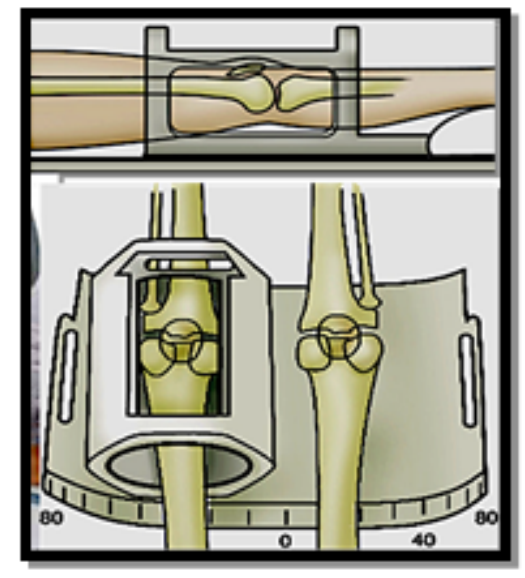

Figure 4: Positioning for MRI Knee joint

\section{Material and Methods}

A population of 20 subjects who presented themselves in Sri Ramachandra Hospital (A tertiary care university hospital in Chennai, India) to the Department of Radiology and Imaging Sciences of either sex whose reports and image data's are collected prospectively during the study period of 3 months (December 2011- February 2012). All the patients' data within the study period were collected. Patients were selected irrespective of their age group, gender and pathologic findings. A detailed history with various patient's data includes patient demography, age, sex and the study reports are also collected and is entered in a specially designed Profoma. 


\subsection{Materials:}

The GE Signa HDx MRI system (1.5T) whole body magnetic resonance scanner with the 8 channel, 9-element phased-array knee coil is used for imaging. The MR imaging sequences most commonly used in the assessment of joint cartilage are 2D or multisection sagittal \& axial (FSE) Proton Density Weighted with Fat Suppression (PD FS), sagittal gradient echo sequence, T2 weighted Fast Spin Echo, T1 weighted (T1w SE)and coronal Shot TAU Inversion Recovery (STIR); In addition to the routine protocol the $\mathbf{T 2}$ Mapping sequence is added.

\subsection{T2 Mapping:}

Routine MR imaging allows a subjective assessment of cartilage T2 changes, whereas quantitative T2 mapping provides objective data by generating either a color or a gray-scale map representing the variations in relaxation time within cartilage. A multiecho SE technique is used to measure T2 values. T2 Map acquires multiple scans at each location; each set of scans has a unique TE, resulting in a data set of images that represent different T2 weighting. The number of TEs per scan (not a selectable parameter) is the scan parameter that determines the number of images that are acquired at each location. For example, if 10 locations are prescribed with 8 TEs per scan, then there are 10 data sets with 8 images per location. Each image within a data set or location has 8 unique T2-weighted images, because all lines of k-space are filled with one TE. The data is processed in Functool, producing T2 color maps to visualize the changes in cartilage that are not visible in gray scale MR images. In MRI T2 decay imaging of hyaline articular cartilage reflects interactions among water molecules and between water molecules and surrounding macromolecules and is highly sensitive to alterations of the cartilage matrix. Increased interactions between water and macromolecules such as those of collagen result in decreased T2. Thus, $\mathrm{T} 2$ is highly sensitive to changes in hydration (or, nearly equivalently, collagen concentration) and in the normally anisotropic orientation of the collagen fibrils within the extracellular cartilage matrix (50) that result from physiologic or pathophysiologic processes in cartilage. In normal cartilage, regional and zonal differences in density and organization of the collagen matrix appear as variations in T2. There is good evidence that T2 mapping is useful for identifying sites of early-stage degeneration (early disruption of the collagen matrix) in cartilage, which appear as areas with $\mathrm{T} 2$ higher than that of normal cartilage.

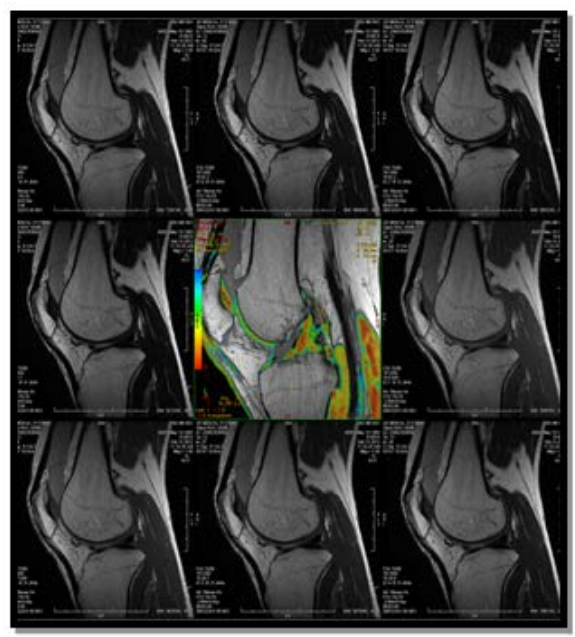

Figure 5: T2 Mapping sequence - T2 images with 8 TEs (ms) - 8.9, 17.8, 28.7, 35.6, 44.4, 53.3, 62.2, 71.1With the generated T2 Color Map (center image) 
Raj Kumar Soundarajan, Rajeswaran R and Sheila Elangovan; Magnetic Resonance T2 Relaxometry In Knee Joint Patellar Cartilage Imaging. Journal of Biomedical Engineering and Medical Imaging, Volume 3, No 5, October (2016), pp 18-28

\subsection{Applications:}

T2 Map is used to image cartilage. Healthy collagen, which has a short T2 relaxation time, is a highlyorganized, anisotropic structure that restricts the flow of water. The body uses this cushion of water to absorb shock. The T2 relaxation time of articular cartilage is a function of the water content of the tissue. An increase in T2 relaxation times within cartilage has been associated with matrix damage, especially loss of collagen matrix. The T2 Map and the parametric images produce visible image contrast changes (T1 and T2 alterations) in early stages of cartilage degeneration, such as osteoarthritis.

Table 1: Protocol for T2 Mapping - Sagittal plane

\begin{tabular}{|c|c|}
\hline \multicolumn{2}{|c|}{ PARAMETERS } \\
\hline Scan plane & Sagittal \\
\hline Mode & $2 \mathrm{D}$ \\
\hline Pulse sequence family & Spin echo \\
\hline Pulse sequence & FSE-T2 \\
\hline TR (ms) & 1000 \\
\hline TE (ms) & $(1) 08.9,(2) 17.8,(3)$ \\
& $26.7,(4) 35.6,(5) 44.4$ \\
\hline & $(6) 53.3,(7) 62.2$ \\
\hline FOV (cm) & $(8) 71.2$ \\
\hline Slice thickness (mm) & $16 \times 16$ \\
\hline Spacing (mm) & 2 \\
\hline Flip angle (degree) & 4 \\
\hline Frequency & 90 \\
\hline Phase & 288 \\
\hline NEX & 224 \\
\hline Scan time & 1 \\
\hline
\end{tabular}

\subsection{Post Processing:}

Functool is software package that allows the analysis of MR data sets for post processing. The images can represent changes in image intensity over time, b-value or gradient orientation (diffusion imaging), or frequency (spectroscopy). Results are in the form of graphs and functional maps that can be saved \& documented.

\subsection{1 : T2 Mapping Functool:}

T2 MAP protocol post processes data sets acquired using the T2Map application. The T2Map acquisition is displayed in Functool where the T2 relaxation time color map is coded to capture T2 values from the TE range of the acquired images.

1. The source image is displayed in the upper left Map viewport

2. The T2Map Preset-1 parametric image is displayed in the lower left viewport.

3. The T2Map Preset-2 parametric map is displayed in the lower right viewport. 
4. A curve displaying signal intensity on the vertical axis and the echo number on the horizontal axis is displayed in the upper right viewport. A data point for each echo is plotted when an ROI is deposited on any of the three images.

T2Map layout is comprised of 4 viewports

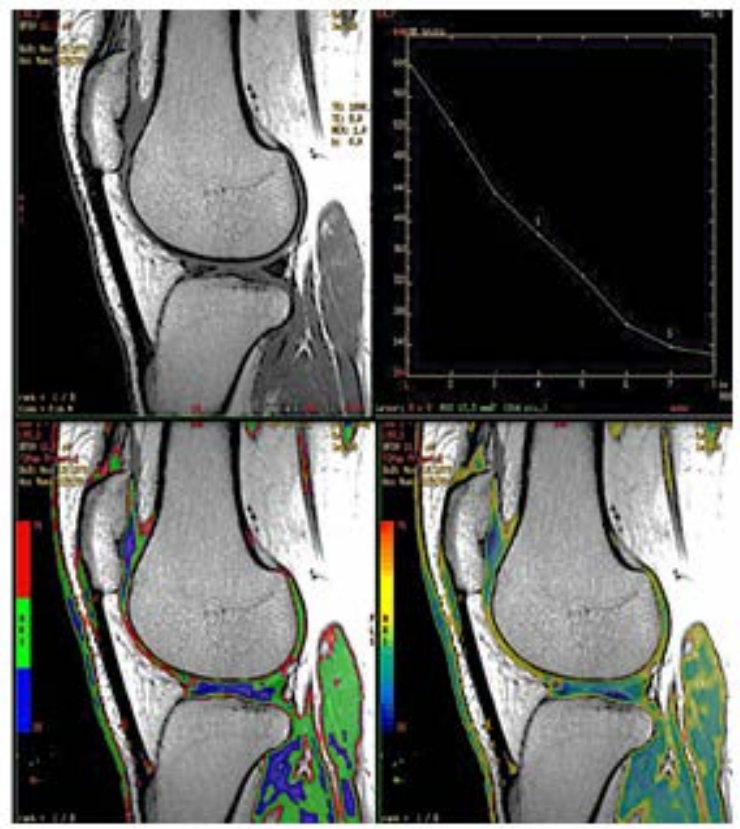

Figure 6: T2 Mapping layout in Functool application (Version: ADW4.4GE workstation)

\subsection{2: T2 Mapping Workflow}

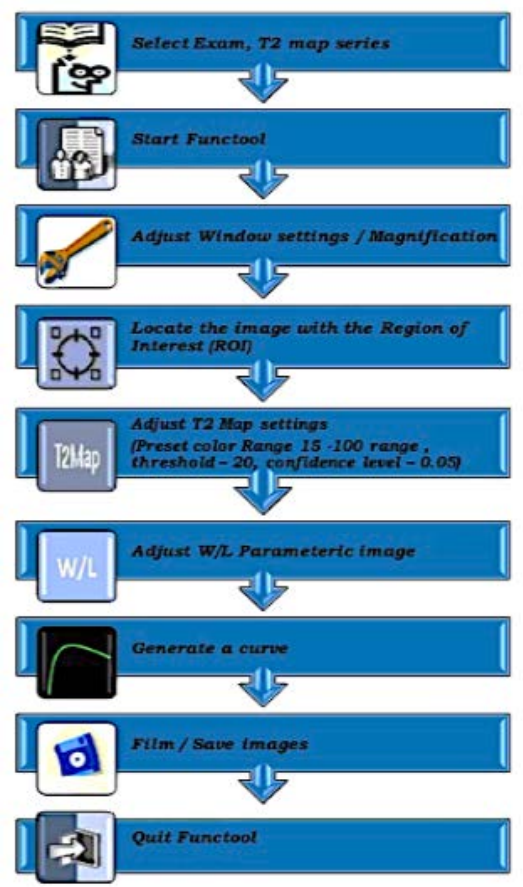


Raj Kumar Soundarajan, Rajeswaran R and Sheila Elangovan; Magnetic Resonance T2 Relaxometry In Knee Joint Patellar Cartilage Imaging. Journal of Biomedical Engineering and Medical Imaging, Volume 3, No 5, October (2016), pp 18-28

\subsection{Interpretation of The Color Mapping:}

The parametric map color scheme is as follows:

- $\quad$ Short $\mathrm{T} 2$ structures $=$ Orange to Red

- Intermediate $\mathrm{T} 2$ structures = Yellow

- $\quad$ Long T2 structures = Green to Blue

\section{Observations and Result}

The post processing of MR Cartigram (Sagittal) of 20 patients on the Functool reveals the following results.

In this study we calculated the T2 relaxation times of the cartilages, and found that there is an increase in those values due to various reasons, and thus commercial MR imaging has been used to confirm the diagnosis. It allows a subjective assessment of cartilage T2 changes, whereas quantitative T2 mapping provides objective data by generating either a color or a gray-scale map representing the variations in relaxation time within cartilage. A multiecho SE technique is used to measure T2 values. T2 Mapping acquires multiple scans at each location; each set of scans has a unique TE, resulting in a data set of images that represent different $\mathrm{T} 2$ weighting.

The data is processed in FuncTool, producing T2 color maps to visualize the changes in cartilage that are not visible in gray scale MR images. In our study,

- Out of 9 patients with the history of trauma there is an increase in the $\mathrm{T} 2$ relaxometry values for 6 patients.

- Out of 7 patients with the history of unknown knee pain shows increase in the T2 relaxometry values for 5 patients.

- Patients with history of knee instability/ pain on action and others (No. of patients 2/1/1 respectively) shows less significant increase in the T2 Relaxation time.

On comparing the T2 Relaxometry values of the patients with the history of Osteoarthritis vs. others, shows an increase in the $\mathrm{T} 2$ relaxation time of the knee joint cartilages. This is due to the early morphological changes occurs due to osteoarthritis in the knee joint cartilages.

There is good evidence that T2 mapping is useful for identifying sites of early-stage degeneration (early disruption of the collagen matrix) in cartilage.

Table 2: Comparison of T2 relaxometry values of Osteoarthritis and other patients

\begin{tabular}{|c|c|}
\hline OSTEOARTHRITIS & 6 \\
\hline OTHERS CONDITIONS & 14 \\
\hline
\end{tabular}




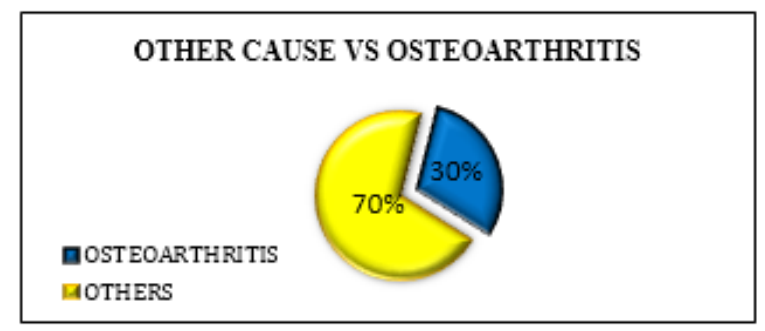

Graph 1: Percentage of T2 relaxometry values of Osteoarthritis over other patients

Table 3: Clinical symptomatology for other patients

\begin{tabular}{|c|c|c|}
\hline $\begin{array}{c}\text { CLINICAL } \\
\text { SYMPTOMS }\end{array}$ & $\begin{array}{c}\text { NO. OF } \\
\text { PATIENTS }\end{array}$ & $\begin{array}{c}\text { PERCENTAGE } \\
(\%)\end{array}$ \\
\hline Trauma & 9 & 45 \\
\hline Rest Pain & 7 & 35 \\
\hline Instability & 2 & 10 \\
\hline Pain On Activity & 1 & 5 \\
\hline POLIO & 1 & 5 \\
\hline
\end{tabular}

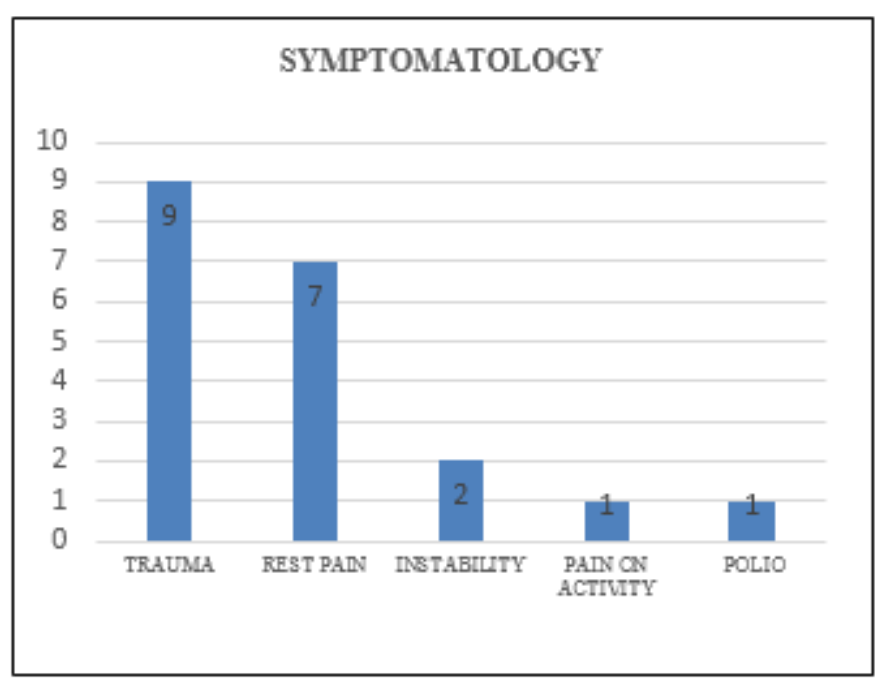

Graph 2: Distribution of other patient's symptomatology

Table 3 shows the clinical symptoms of the patients who came for MRI Knee Joint

This graph shows that $45 \%$ of the patients are with the history of trauma, followed by rest pain that is around $35 \%$, then with the knee instability $10 \%$, and with least symptoms of pain on action \& with polio disorder of $5 \%$ each 


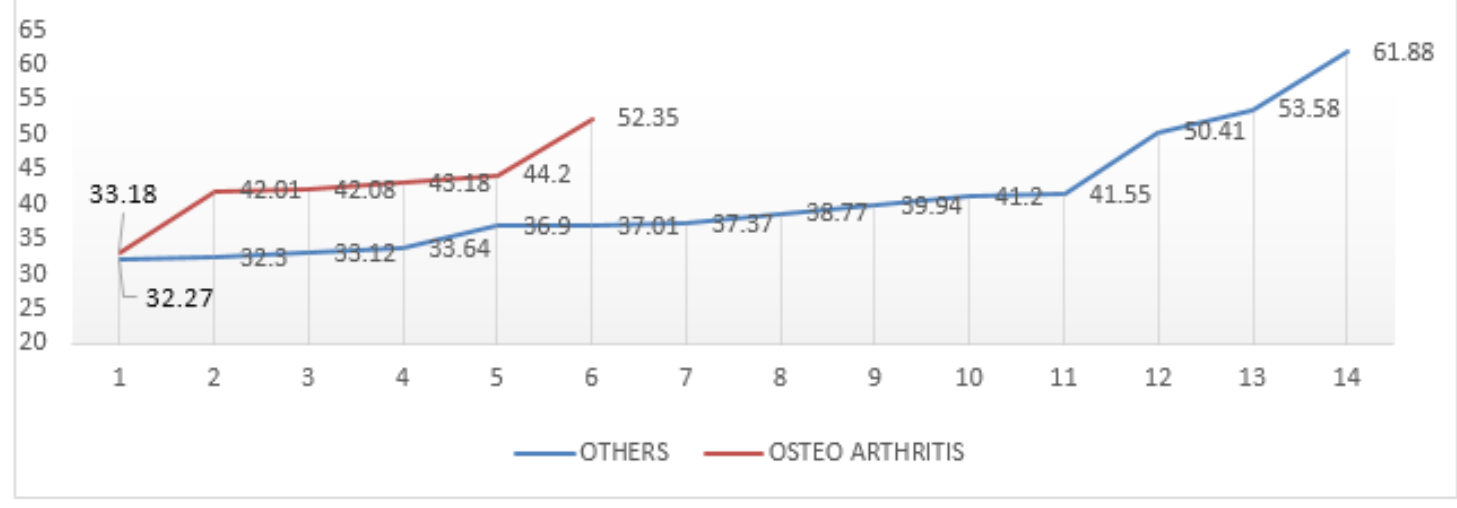

Graph 3: Comparison of T2 relaxometry values of the medial Patellar Cartilage in osteoarthritis patients and in other symptoms.

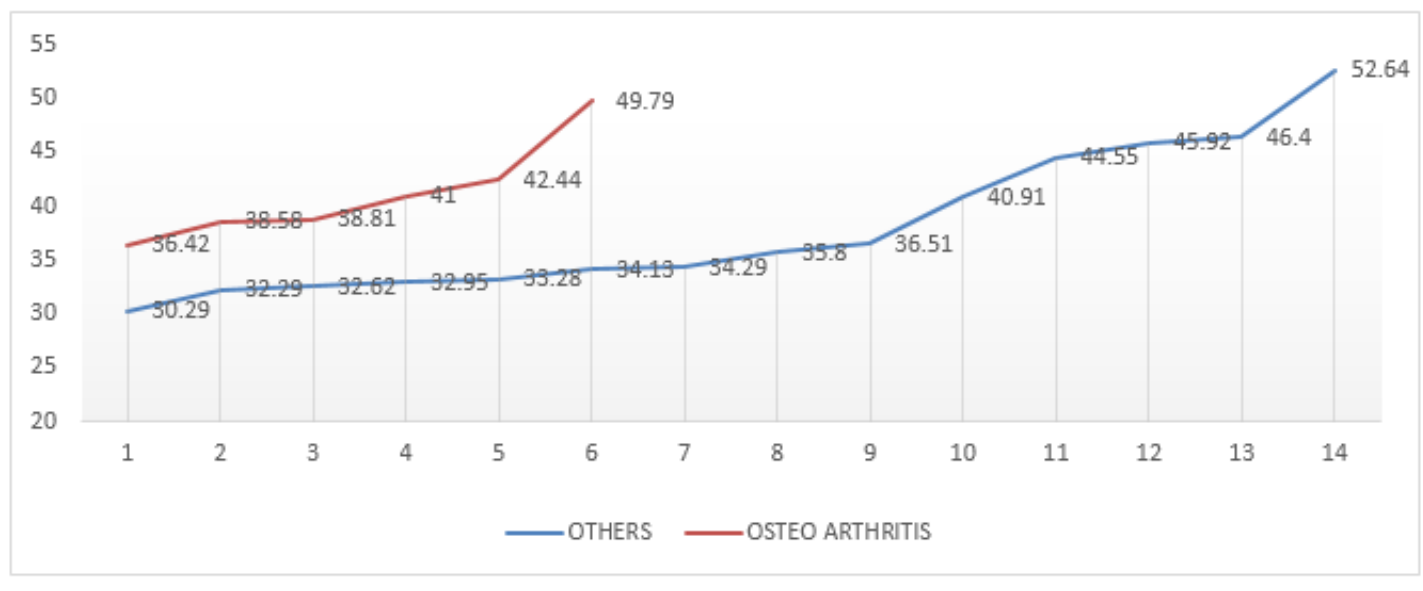

Graph 4: Comparison of T2 relaxometry values of the lateral Patellar Cartilage in osteoarthritis patients and in other symptoms.

\section{Conclusion}

Cartilage edema following trauma (or) Due to osteoarthritis can be picked up early by MR Cartigram. Conventional MRI may not show early cartilage changes hence T2 Mapping is useful in patient management. T2 mapping can be implemented relatively on most clinical MR imaging systems, as pulse sequences for obtaining quantitative T2 maps and software for generating colour T2 maps are now available in commercial packages.

\section{REFERENCES}

[1] Crema MD, Roemer FW, Marra MD, Burstein D, Gold GE, Eckstein F, Baum T, Mosher TJ, Carrino JA, Guermazi A. Articular cartilage in the knee: current MR imaging techniques and applications. Radiographics - .Jan-Feb 2011.

[2] Sharmila Majumdar and Blumenkrantz on Quantitative Magnetic Resonance Imaging Of Articular Cartilage In Osteoarthritis. 
[3] C. Liess, S. Lu“Sse, N. Karger, M. Heller and C.-C. Glu“Er - On the detection of changes in cartilage water content using MRI T2-Mapping.

[4] Tallal C. Mamisch MD, Siegfried Trattnig MD, Sebastian Quirbach MD, Stefan Marlovits MD, Lawrence M. White MD, Goetz H. Welsch, on the Quantitative T2 Mapping of knee cartilage: Differentiation of healthy control cartilage and cartilage repair tissue in the knee with unloading.

[5] Jinfa Xu, Guohua Xie, Yujin Di, Min Bai, Xiuqin Zhao on The Value Of T2-Mapping and DWI in the Diagnosis Of early Knee cartilage injury.

[6] Garry E. Gold, Christina A. Chen,Seungbum, Brian A. Hargreaves, Neal K. Bangerters on Recent Advances in MRI of Articular Cartilage.

[7] Klaus M. Friedrich, Timothy Shepard, Valesca Sarkis De Oliveira, Ligong Wang, James S. Babb, Mark Schweitzer, Ravinder Regatte on the T2 measurements of cartilage in osteoarthritis patients with meniscal tears.

[8] Iwan Van Breuseghem, , Hilde T. C. Bosmans, , Luce Vander Els, Frederik Maes, on the Feasibility of T2 Mapping of human femoro-tibial Cartilage With Turbo Mixed MR Imaging at $1.5 \mathrm{~T}$.

[9] Catherine Westbrook MRI IN PRACTICE - Third Edition.

[10] Alfred L. Horowitz, MRI Physics for Radiologist - Second Edition.

[11] Gray's Anatomy for students - Second Edition. 